\title{
Intrathecal/Intraventricular Linezolid in Multidrug-Resistant Enterococcus faecalis Ventriculitis
}

\author{
Brian F. Lich ${ }^{1}$ Andrew K. Conner ${ }^{2}$ Joshua D. Burks² \\ ${ }^{1}$ Department of Internal Medicine, University of Oklahoma Health \\ Sciences Center, Oklahoma City, Oklahoma, United States \\ 2 Department of Neurosurgery, University of Oklahoma Health \\ Sciences Center, Oklahoma City, Oklahoma, United States
}

\author{
Chad A. Glenn² Michael E. Sughrue ${ }^{2}$
}

J Neurol Surg Rep 2016;77:e160-e161.

\begin{abstract}
Address for correspondence Andrew K. P. Conner, MD, Department of Neurosurgery, The University of Oklahoma Health Sciences Center, 1000 North Lincoln Boulevard, Suite 400, Oklahoma City, OK 73104, United States (e-mail: andrew-conner@ouhsc.edu).
\end{abstract}

\begin{abstract}
Keywords

- intrathecal

- ventriculitis

- linezolid

- external ventricular drain

Background The use of intrathecal antibiotic therapy for the treatment of ventriculitis and/or meningitis has demonstrated efficacy especially when sterilization of the cerebrospinal fluid is not possible with intravenous antibiotics alone.

Case Description We describe the successful treatment of Enterococcus faecalis ventriculitis utilizing intrathecal linezolid in a 32-year-old female patient with severe allergy to vancomycin, prohibitive bacterial susceptibilities, and failure of previous attempts to sterilize the cerebrospinal fluid despite multimodal treatment.

Conclusion Intrathecal linezolid is a useful treatment in the setting of multidrugresistant bacterial ventriculitis. We present a useful dosing regimen for the administration of intrathecal linezolid.
\end{abstract}

\section{Introduction}

Intrathecal (IT) antibiotics have been increasingly utilized for neurosurgical patients with ventriculitis and meningitis. The indication for such use of antibiotics often occurs in the setting of inability to sterilize cerebrospinal fluid (CSF) despite treatment with systemic therapy. The efficacy and safety of such antibiotic administration have been well described over the years, especially in the cases of vancomycin and gentamicin. ${ }^{1}$ However, the use and safety profile of newer generation antibiotics via an IT route remains relatively uncertain. Here, we present a case of a 32-year-old female patient with Enterococcus faecalis ventriculitis who was successfully treated with IT linezolid in addition to systemic antibiotic therapy.

\section{Case Report}

In August 2014, a 31-year-old female patient was originally diagnosed with glioblastoma and subsequently underwent surgical resection via a left frontal craniotomy. Approximately 6 months later, the patient developed wound dehiscence and surgical site infection. The initial antibiotic regimen included vancomycin for which the patient developed a severe allergic reaction. During this time period, the patient underwent multiple wound washouts and revisions though she repeatedly presented back to the hospital with fever and drainage from her cranial incision. Eventually, after developing hydrocephalus, the patient had an external ventricular drain (EVD) placed in April 2015. Shortly thereafter, she was started on therapy with intravenous aztreonam for CSF cultures growing Klebsiella pneumoniae. Repeat cultures additionally grew Candida albicans for which intravenous voriconazole was added to the antimicrobial regimen. Despite this, the patient continued to have surgical site drainage and intermittent fevers. Weeks later, further repeat cultures grew extended spectrum $\beta$-lactamase producing Klebsiella and nonvancomycin-resistant $E$. faecalis as aztreonam was switched to imipenem for appropriate coverage based on sensitivity profiles of the above microbes.
License terms Stuttgart - New York

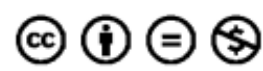

April 26, 2016 accepted after revision August 19, 2016
DOI http://dx.doi.org/ 10.1055/s-0036-1593439. ISSN 2193-6358. 
Again, despite the above therapy, the patient continued to experience marked confusion with fevers and purulent drainage from the surgical site. The patient underwent rotational flap coverage of her surgical site. In addition, the hospital was experiencing a shortage in imipenem for which the patient was transitioned to meropenem. Of note, oral linezolid $600 \mathrm{mg}$ twice daily was also added to the regimen to provide further coverage for the $E$. faecalis considering the lower efficacy of meropenem (vs. imipenem) and the patient's history of severe allergic reaction to vancomycin and cephalosporins. Over the next week, the patient had minimal clinical change as CSF cultures drawn every 48 hours failed to show clearance of the E. faecalis, despite ventricular catheter exchange. Considering the persistence of infection, the decision to add IT linezolid was made given that sensitivities demonstrated antibiotic resistance to gentamycin. IT linezolid was thus started and administered via the EVD at $10 \mathrm{mg}$ daily at a concentration of $2 \mathrm{mg} / \mathrm{mL}$.

After 6 days of adding IT linezolid, the patient showed sterilization of her CSF. From a clinical perspective, 10 days into her course of IT linezolid, the patient began to regain some physical and mental function, prompting removal of the EVD and ventriculoperitoneal shunt placement. At this time, the IT linezolid was discontinued (after receiving 15 days of total IT therapy) though the systemic antibiotics remained. Postoperatively, the patient continued to show stability and improvement, and she was discharged to a rehabilitation facility without complication.

The patient remained stable and was doing well until 3 months later when she returned to the hospital with worsening confusion and recurrent tonic-clonic seizures. Workup at that time revealed intracranial hemorrhage associated with significant recurrent tumor burden and midline shift. Considering the degree of the patient's symptoms and overall poor prognosis, the patient was made comfort care at that time and discharged home under the guidance of hospice.

\section{Discussion}

IT antibiotics have increasingly been shown to be an effective and safe method of drug delivery in neurosurgical cases, especially in those involving antibiotics that do not achieve good central nervous system penetration and in cases wherein CSF sterilization proves to be difficult. Our case here presents the use of IT linezolid, which has not been previously well described.

Linezolid is often considered as a therapeutic option in cases of methicillin-resistant Staphylococcus aureus and vancomycin-resistant E. faecalis (VRE), where other antibiotics may not be tolerated based on side-effect profiles. Situations of VRE meningitis have previously been described in multiple case reports and include treatment via both systemic options (primarily with linezolid or daptomycin) and IT options (quinupristin-dalfopristin, chloramphenicol, daptomycin). ${ }^{2-4}$ Despite this, there is a paucity of data regarding the use of IT linezolid.
The ideal situation for using IT linezolid remains unknown. Earlier reports of central nervous system (CNS) penetrance of linezolid are widely variable, ranging from 28 to $70 \%{ }^{5,6} \mathrm{~A}$ better understanding of the drug's action in the CNS following systemic administration is necessary to elucidate benefits of administering the drug IT.

As presented in our case, a patient with multiple antibiotic allergies and an EVD-related E. faecalis CSF infection that does not clear with prolonged use of systemic linezolid (8 days), demonstrating antibiotic resistance to gentamycin, and despite ventricular catheter exchange (excluding the possibility of bacterial colonization), may serve as a good candidate for the use of IT linezolid. Here, the use of IT linezolid did show rapid clearance of $E$. faecalis and sterilization of the CSF within 6 days of initiation. From a clinical perspective, there were no immediately apparent adverse reactions to the IT linezolid, as the patient was believed to have cleared her infection. However, considering the patient's intracranial bleed associated with tumor progression/ recurrence and death 3 months later; it is difficult to fully evaluate the true impact and efficacy of this treatment. Despite this difficult outcome, we hope this case presents an example of the use of IT linezolid and how it may be dosed and serve as a mechanism for CSF sterilization in difficult neurosurgical infections in the future.

Conflicts of Interest

The authors listed report no conflict of interest.

\section{Disclosures}

None.

Funding

This report has no specific funding sources.

\section{References}

1 Remeš F, Tomáš R, Jindrák V, Vaniš V, Setlík M. Intraventricular and lumbar intrathecal administration of antibiotics in postneurosurgical patients with meningitis and/or ventriculitis in a serious clinical state. J Neurosurg 2013;119(6):1596-1602

2 Wang JS, Muzevich K, Edmond MB, Bearman G, Stevens MP. Central nervous system infections due to vancomycin-resistant enterococci: case series and review of the literature. Int J Infect Dis 2014; 25:26-31

3 Denetclaw TH, Suehiro I, Wang PK, Tolliver GL. Successful treatment of ventriculostomy-associated meningitis caused by multidrug resistant coagulase-negative Staphylococcus epidermidis using low-volume intrathecal daptomycin and loading strategy. Ann Pharmacother 2014;48(10):1376-1379

4 Knoll BM, Hellmann M, Kotton CN. Vancomycin-resistant Enterococcus faecium meningitis in adults: case series and review of the literature. Scand J Infect Dis 2013;45(2):131-139

5 Bain KTWE, Wittbrodt ET. Linezolid for the treatment of resistant gram-positive cocci. Ann Pharmacother 2001;35(5):566-575

6 Gill CJMM, Murphy MA, Hamer DH. Treatment of Staphylococcus epidermidis ventriculo-peritoneal shunt infection with linezolid. J Infect 2002;45(2):129-132 